\title{
Self-help smoking cessation interventions in pregnancy: a systematic review and meta-analysis
}

\author{
Felix Naughton, A. Toby Prevost \& Stephen Sutton \\ General Practice and Primary Care Research Unit, Institute of Public Health, University of Cambridge, UK
}

\section{ABSTRACT}

Aims Self-help smoking cessation interventions for pregnant smokers are of importance due to their potential to be wide-reaching, low-cost and their appeal to pregnant smokers who are interested in quitting smoking. To date, however, there has been no systematic assessment of their efficacy. This systematic review aimed to assess the efficacy of self-help interventions for pregnant smokers and to investigate whether self-help material intensity, type or delivery are associated with cessation. Methods The literature was searched for randomized and quasi-randomized controlled trials of self-help smoking cessation interventions for pregnant smokers without significant cessation counselling. Fifteen trials met the inclusion criteria and relevant data were extracted independently. Results The primary metaanalysis pooled 12 trials comparing usual care (median quit rate 4.9\%) with self-help (median quit rate 13.2\%) and yielded a pooled odds ratio (OR) of 1.83 [95\% confidence interval (CI) 1.23-2.73], indicating that self-help interventions on average nearly double the odds of quitting compared with standard care. However, a further meta-analysis failed to find evidence that intervention materials of greater intensity increase quitting significantly over materials of lesser intensity (pooled OR $=1.25,95 \%$ CI 0.81-1.94). There was insufficient evidence to determine whether the tailoring of materials or levels of one-to-one contact were related to intervention efficacy. Conclusions Self-help interventions appear to be more effective than standard care although, due mainly to a lack of trials, it is unclear whether more sophisticated and intensive approaches increase intervention effectiveness.

Keywords Meta-analysis, pregnancy, review, self-help, smoking cessation, tailoring.

Correspondence to: Felix Naughton, Institute of Public Health, University of Cambridge, Forvie Site, Cambridge CB2 OSR, UK.

E-mail: fmen2@medschl.cam.ac.uk

Submitted 7 June 2007; initial review completed 17 September 2007; final version accepted 13 December 2007

\section{INTRODUCTION}

In addition to damaging the health of the mother, prenatal smoking is associated consistently with a number of complications during pregnancy [1,2] and several postnatal conditions affecting the infant [2]. Considering that between $17 \%$ [3] and $27 \%$ [4] of pregnant women are estimated to smoke throughout pregnancy in the United Kingdom, it is of high importance that costeffective interventions are developed and disseminated so that smoking-related health complications can be avoided.

While face-to-face smoking cessation behavioural support is generally available for pregnant smokers, attendance rates are low [5]. One potentially attractive alternative for pregnant women who do not wish to receive face-to-face advice, in some cases because of fear of self-disappointment or of being judged [6], is self-help. Self-help smoking cessation interventions are of particular interest in the smoking cessation field as they are generally low-cost and have the potential to be widereaching. While booklets are the most common form of self-help materials, they can also include video, telephone (recorded messages) and computer-based systems.

A recent Cochrane review concluded that, compared with receiving no materials, self-help materials on their own increase cessation rates only marginally among non-pregnant smokers [7]. Tailored self-help materials, however, were found to increase quitting significantly compared with control groups given mainly standard materials, with a pooled odds ratio (OR) of 1.42 [95\% confidence interval (CI) 1.26-1.61]. Tailoring refers to the use of participant characteristics to individualize intervention materials and is believed to be effective 
because it increases the relevance of the information to the individual and attentiveness to the message [8].

Self-help materials are common components in behavioural interventions for pregnant smokers and are considered to be important adjuncts to cessation counselling to help pregnant smokers quit smoking [9]. In addition, they have been identified as being of high interest to this group of smokers [10]. They also reduce the need for detailed cessation advice to be provided by antenatal health-care professionals, who are regarded by some as being ineffective at doing so $[11,12]$. However, it is unclear whether self-help approaches on their own can increase quitting among pregnant smokers.

Several reviews confirm that interventions promoting smoking cessation during pregnancy can reduce smoking rates significantly [13-15]. Lumley et al.'s review [14], the largest systematic review in this area to date, included trials providing a variety of cessation interventions, including self-help materials, counselling techniques, bio-feedback, social support, financial incentives and nicotine replacement therapy. Lumley et al.'s meta-analysis revealed a relative risk of 0.94 (95\% CI 0.93-0.95), indicating that the risk of smoking in late pregnancy was significantly lower among intervention groups (median quit rate $13.4 \%$ ) than usual care groups (median quit rate $8.5 \%$ ). When converted into the odds of quitting, the OR is 1.67 (95\% CI 1.43-1.95), favouring the intervention groups. They also found that interventions of greater intensity, determined mainly by levels of personal contact, added little benefit. Kelley et al. [13] reported similarly that the level of counselling provided within an intervention was not associated with efficacy.

Although Lumley et al. broadly assessed the efficacy of different cessation strategies, most trials fell into either cognitive-behavioural, stages-of-change or 'other' categories, with no breakdown by intervention method. As far as the authors of this review are aware, there have been no systematic reviews assessing the efficacy of specific intervention approaches for smoking cessation in pregnancy.

Considering the potential benefits of self-help already identified and their frequent use in smoking cessation interventions for pregnant smokers, it is important to determine whether they can be effective on their own for this target group. Additionally, increasing our understanding of whether the intensity or type of materials or method of delivery is associated with efficacy can inform future intervention development.

\section{Objectives}

This review has five research questions referring to selfhelp smoking cessation interventions for pregnant smokers and their impact on smoking rates in pregnancy.
Primary focus

1 Do self-help interventions increase quit rates compared with usual care?

\section{Subgroup analyses}

2 Do booklet-based self-help interventions increase quit rates compared with usual care?

3 Is the quantity of face-to-face contact associated with intervention efficacy?

\section{Secondary focus}

4 Are self-help materials of a greater intensity more efficacious than those of lesser intensity?

\section{Subgroup analysis}

5 Are tailored self-help interventions more efficacious than non-tailored self-help interventions?

\section{METHOD}

\section{Study selection and characteristics}

\section{Study design and participants}

Controlled trials with a randomized or quasi-randomized allocation including pregnant smokers at any stage of care aged 16 years and over were considered. Trials were not included if the sample consisted only of pregnant quitters or those with a history of substance misuse.

\section{Intervention}

Trials were considered if at least one of the experimental arms met the self-help definition. The definition used, based on Lancaster \& Stead's [7] description, defines selfhelp as the provision of structured materials that assist the individual in making a quit attempt and sustaining abstinence without significant assistance from a health professional or group support. Experimental arms that provided smoking cessation counselling in addition to that received by the control arm were not counted as self-help. Contact for the purposes of introducing the programme or materials or to ensure the proper grasp of materials during either initial or follow-up contact was acceptable. While no restriction was set regarding the quantity of contact time, any trials that provided more than 15 minutes of contact time in total were removed from the main analysis as part of a sensitivity analysis.

Self-help can include written, video, audio-based, telephone (recorded messages) or computer-based materials. Interventions with organized social support components such as buddying, those that contained financial incentives or those that provided only brief leaflets focusing on 
the harm of smoking during pregnancy and offering only basic tips for smoking cessation were not considered to be self-help.

\section{Outcome measures}

For analysis purposes, the latest biochemically validated point prevalence smoking status measure taken in pregnancy was the preferable outcome measure, as it is the most typical in this area and therefore aids comparability between trials. Self-report only and postpartum outcomes were acceptable.

\section{Search strategy}

Four major bibliographic databases were searched in February 2006: MEDLINE, EMBASE, PsycINFO and CENTRAL, with no restrictions on publication date or language. Highly sensitive search strategies were used for MEDLINE [16], and EMBASE and PsycINFO [17] in combination with the keywords 'pregn*', 'smok*', 'tobacco', 'cessation', 'quit*' and other appropriate synonyms. For CENTRAL, keywords only were used. Bibliographies of relevant reviews and published studies were hand-searched.

\section{Quality assessment}

Methodological quality assessment was carried out using Lumley et al.'s [14] quality criteria based on recommendations in the Cochrane Reviewer's Handbook 4.1.5 [18], although for this review a partial rather than full intention-to-treat approach criterion was used (see quality criteria 5). Quality was assessed using the following criteria:

1 Selection bias: allocation concealed at least until the point of allocation and methods of concealment are robust against clinician and participant bias. Allocation carried out using a true randomization method and not pseudo-randomization.

2 Performance bias: caregiver blinded to allocation.

3 Detection bias: smoking status assessed using biochemical validation.

4 Sample size justification is given.

5 Attrition bias: all participants who drop out are considered smokers, not including those withdrawn from the study by the researchers for either having a pregnancy related complication, e.g. miscarriage or abortion, or having moved antenatal care providers (partial intention-to-treat).

If all the above criteria are met, the study is described as having a low risk of bias. If three or four criteria are met then a moderate risk of bias is assumed, otherwise a high risk of bias is assumed.

\section{Data extraction}

All abstracts located by the search strategy were examined independently by two reviewers (F.N. and S.S.). Studies that could not be excluded by their title or abstract were obtained in full text and read. Reviewers then discussed which studies should be included; any disagreements were discussed until a consensus was reached. All data were extracted independently by the two reviewers. Discrepancies were resolved through discussion and referral to the text of the studies and by contacting the authors of the study.

\section{Quantitative data synthesis}

Measures of treatment effect and unit of analysis issues

The OR was chosen as the measure of effect due to its advantageous mathematical properties over the relative risk [19]. As two trials observed zero percentage group quit rates, a value of 0.5 was added to all cells within the $2 \times 2$ table to enable ORs to be calculated [19]. The two cluster-randomized trials included in the review [20,21] were adjusted according to Moore et al.'s intracluster correlation coefficient (ICC), as Lawrence et al. did not report an ICC. As these trials used different units of randomization, sensitivity analyses were carried out adjusting the ICC to a high and a low extreme in order to ensure that the approximation was robust [22].

\section{Assessment of heterogeneity and reporting biases}

Visual inspection of the forest plot from the main metaanalysis, the $\chi^{2}$ test for heterogeneity and the $\mathrm{I}^{2}$ value [23] were used to assess the level of heterogeneity across the trials. A funnel plot was examined to investigate whether there was a systematic difference between the results of small and large studies.

\section{Data synthesis}

As some level of clinical heterogeneity was expected, as behavioural interventions tend to vary in content and success of implementation, a random effects model was adopted for the meta-analyses estimating intervention effects with 95\% CI and significance at the 5\% level.

\section{Analyses}

To address the primary research question, a metaanalysis was carried out pooling trials that compared a self-help intervention with usual care. Where there was more than one self-help experimental arm, the arm of greater intensity or the one hypothesized to be more efficacious was used for analysis. To address the second research question, a meta-analysis was carried out on a subgroup of trials that compared self-help booklet arms 
to usual care. In order to address the third research question, all trials included in the primary meta-analysis were dichotomized by level of face-to-face contact and a relative OR was calculated comparing the two subgroups on their relative efficacy. Five minutes was chosen as a cut-off between brief and extended face-to-face contact based on the conclusions of an expert panel [9].

To address the fourth research question, a metaanalysis was carried out pooling trials that compared two self-help arms of differing material intensity. Arms that received additional self-help materials to those received in another trial arm, or those receiving more detailed, targeted or interactive self-help materials were considered to be the more intensive. Finally, to address research question 5, a meta-analysis of trials comparing tailored and non-tailored intervention arms was carried out.

\section{Sensitivity analyses}

Sensitivity analyses were carried out excluding trials that recruited pregnant quitters at baseline, and did not separate them from smokers for analysis [21,24], that provided more than 15 minutes of contact time [20], and included either clinical reinforcement or where it was difficult to determine whether face-to-face contact included any smoking cessation counselling [25-27]. These 'borderline trials' were removed from analyses in order to establish whether they introduced bias [28]. Additionally, a sensitivity analysis was carried out removing one trial where it was difficult to determine whether control materials should be classified or not as self-help [29], and a further analysis removing all trials that used self-report measures only to establish smoking status.

\section{Study characteristics}

Of the 15 trials that met the inclusion criteria, 12 compared either one or two self-help arms with a usual care control arm and three compared a self-help control arm with a self-help experimental arm (see Table 1).

\section{Primary focus: usual care versus self-help}

Usual care consisted generally of routine advice to quit smoking and the provision of brief written materials; only one trial reported giving no advice or materials to the control group [30]. The self-help arms can be broken down into six booklet interventions [21,26,30-33], two video interventions [34,35] and four interventions that were booklet-based but with additional components: a computer-tailored program [20]; an audiocassette [36]; written prescriptions and letters of encouragement from health professionals [25]; and medical letters, a 'buddy' advice letter and tipsheet, a quarterly newsletter and some additional information leaflets [27].
Six self-help interventions were classified as providing either brief advice ( $\leq 5$ minutes) $[21,31,35]$ or no faceto-face contact, as materials were sent by post $[33,36]$ or provided by hand without instruction [32]. The other six self-help interventions were classified as providing extended contact (>5 minutes). This included one booklet intervention [30], which was described by the authors as most probably providing more than 5 minutes of face-to-face contact (Valbo A, personal communication, 21 July, 2006).

All but one trial identified above [35] included a usual care and self-help booklet arm and so were used to compare usual care with self-help booklets.

\section{Secondary focus: self-help versus more intensive self-help}

The remaining three trials of the 15 identified compared a self-help booklet to tailored self-help guides [24], basic self-help materials to the same materials plus six self-help videos [29] and a self-help booklet to the same booklet plus a tailored interactive telephone system [37]. Two of these trials provided the more intensive experimental arm materials by post [24,29]; the other provided an information brochure about the telephone system by post and a 10-minute phone call instructing participants on its use [37].

Four of the trials described as usual care versus selfhelp included comparisons of two self-help arms of differing intensity; two trials comparing booklet interventions $[26,30]$, one comparing a booklet with a video intervention [34] and one comparing a booklet intervention with the same booklets plus a computer-tailored program [20]. In total, seven trials were included in this secondary analysis.

Three of the trials included in the secondary analysis incorporated theoretically underpinned tailored systems or materials based primarily on the Transtheoretical Model (TTM) [38], and compared them with a nontailored self-help comparison arm. Ershoff et al. [37] provided a tailored interactive telephone system providing stage-appropriate custom messages, Strecher et al. [24] provided tailored smoking cessation guides and Lawrence et al.'s [20] computer-tailored program provided onscreen, audio and printed feedback. For Ershoff et al. and Lawrence et al. tailored materials were received in addition to the non-tailored self-help manuals.

\section{Sample characteristics}

Across all trials reporting sample characteristics, the median proportion of primiparous participants was $38.5 \%$ [interquartile range (IQR) 33.1-44.4\%], the median age was 26.4 years (IQR 23.6-28.0), the median number of cigarettes smoked per day at recruitment was 


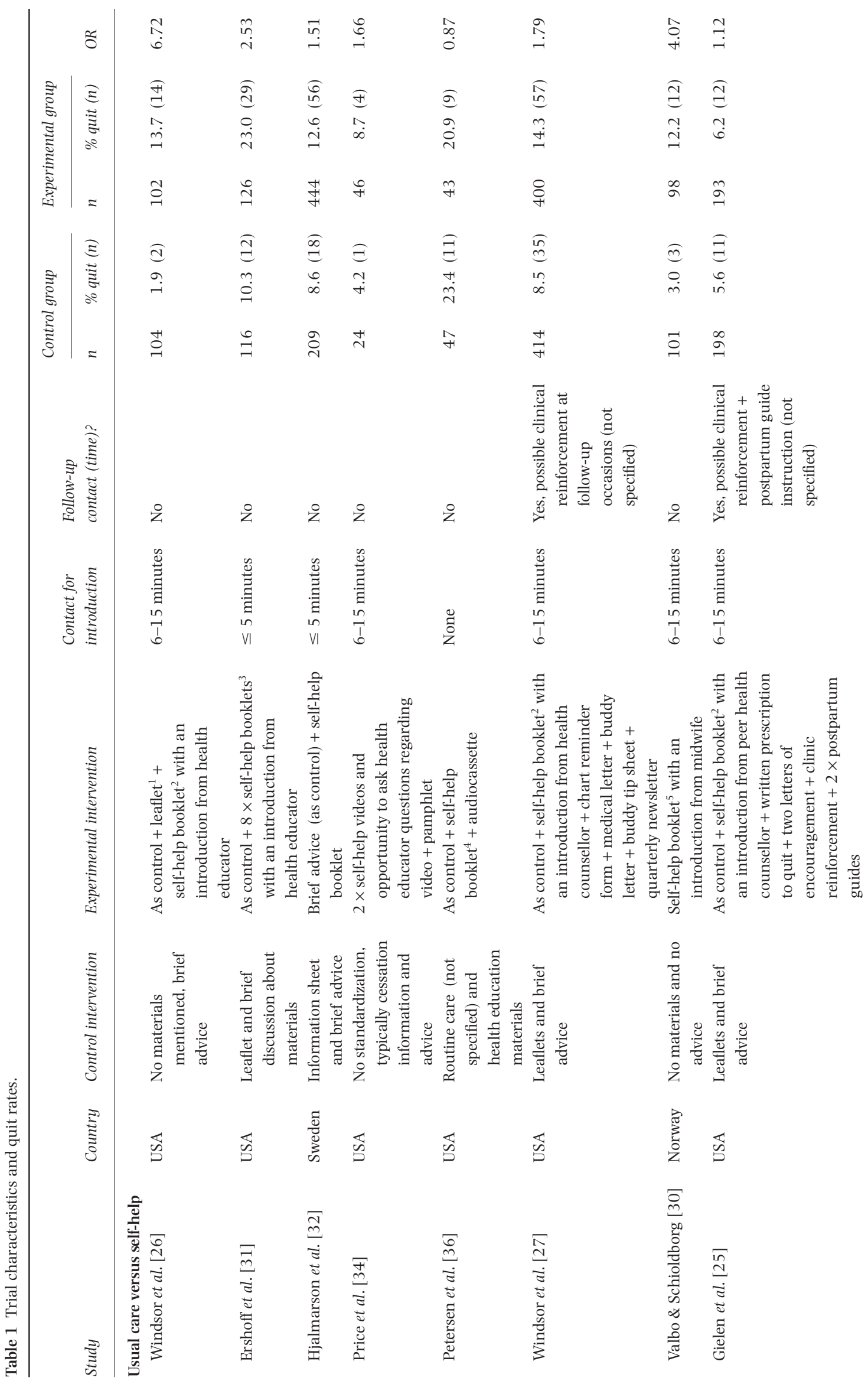




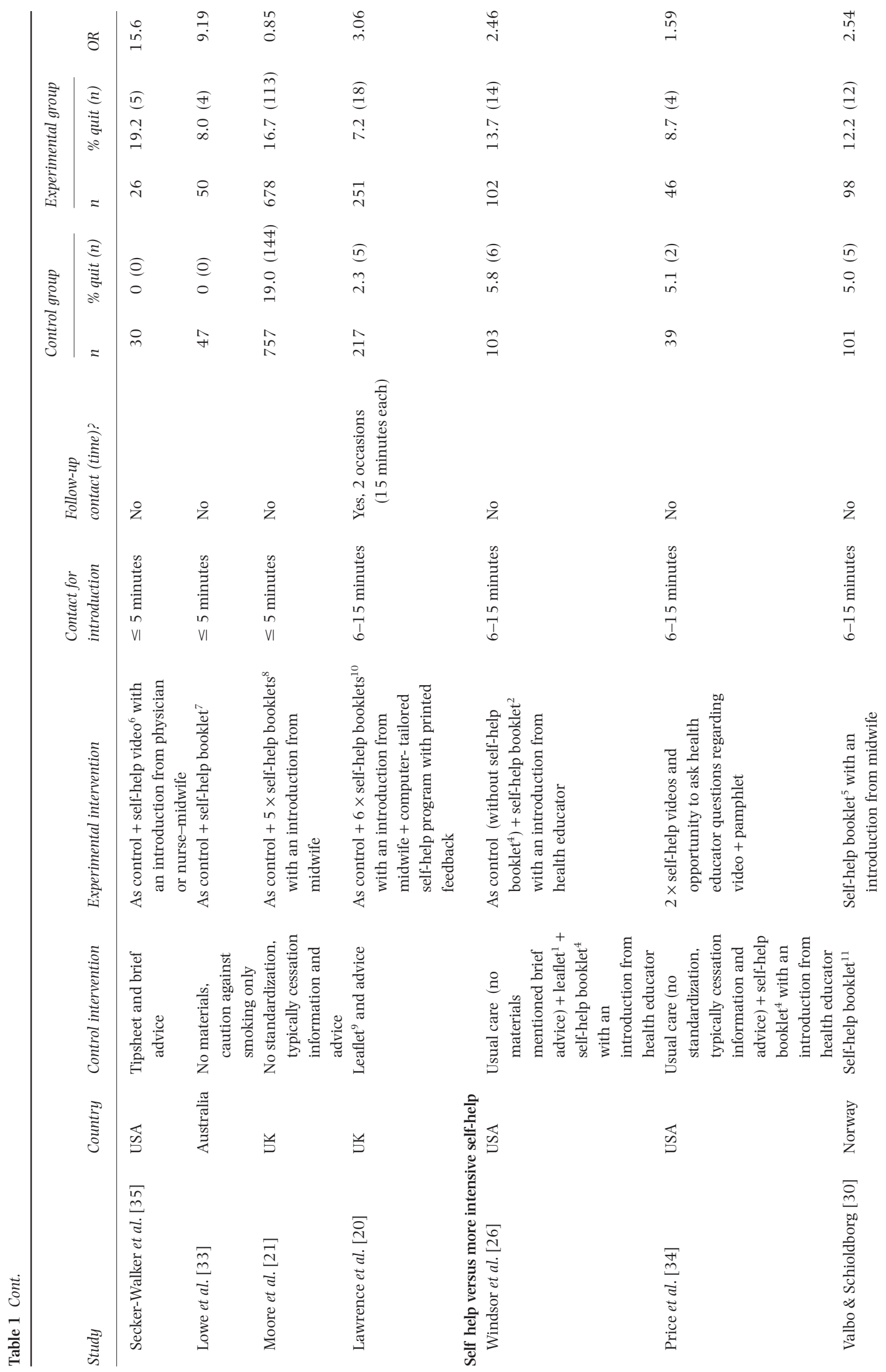




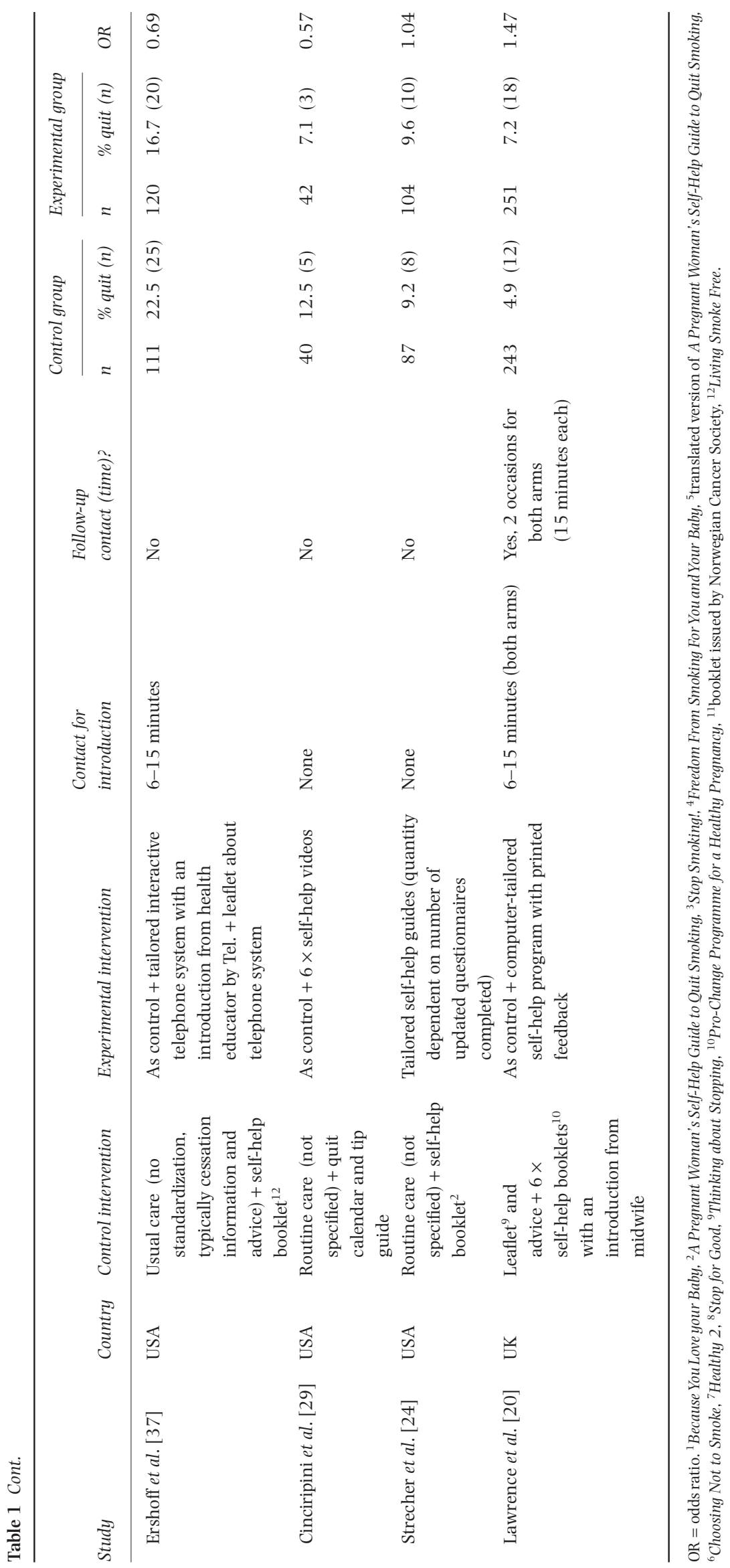


9.1 (IQR 6.3-11.9) and the median proportion of white participants was 70.0\% (IQR 48-84.3\%).

\section{Outcomes}

Approximately, the length of the research period ranged from 6 weeks to 28 weeks with a mean length of around 16 weeks. Twelve trials assessed smoking outcomes either late in the second or during the third trimester. The remaining three trials determined smoking status either during late pregnancy or postpartum $[24,26,30]$. The majority of trials assessed the point prevalence of smoking and biochemical validation methods and cutoffs were satisfactory.

\section{Methodological quality}

Overall, five trials were classified as having a medium risk of bias [20,21,26,27,37] and the remaining 10 as having a high risk of bias (see Table 2).

Only two trials gave sufficient description to determine that adequate randomization and allocation concealment procedures had been carried out [20,21], and only three trials described blinding the caregiver to treatment group allocation [31,36,37]. The majority of trials validated smoking status biochemically, leaving only three trials relying upon self-report alone [24,30,36]. Six of 15 trials reported a formal sample size calculation.

For meta-analytical purposes, it was not possible to adopt a partial intention-to-treat approach for five trials $[20,30,32,34,36]$, due to the exclusion of participants from the analysis who were randomized to the trial arms but who later declined to participate or who were lost to follow-up.

The overall bias classification was deemed inappropriate for use for analysis because many trials provided insufficient description of allocation and blinding procedures and as a partial intention-to-treat approach was able to be adopted for several trials, even though they themselves had not done so $[21,33,35]$. However, the impact of using self-report to determine smoking status was assessed as part of a quality component approach [39].

\section{RESULTS}

Figure 1 summarizes trial flow. The total number of participants included in the primary and secondary metaanalyses were 4721 and 1487 , respectively.

Primary focus: usual care versus self-help

The median quit rates in the usual care and self-help groups of the 12 trials were $4.9 \%$ and $13.2 \%$, respectively, and the IQR of ORs between groups across the studies was 1.22-6.06. The pooled OR from the random effects meta-analysis indicates that self-help interventions increased quit rates significantly over usual care (pooled OR $=1.83,95 \%$ CI 1.23-2.73) (Fig. 2). This equates to an absolute difference between groups of approximately $5 \%$.

Heterogeneity statistics indicate that this set of trials are significantly heterogeneous $(Q=28.89, P=0.002$; $\mathrm{I}^{2}=61.9 \%$ ). Additionally, a funnel plot indicated some asymmetry at the small study end, with the smaller studies showing greater intervention effects.

A subgroup analysis of 11 trials found self-help booklet-based interventions more efficacious than usual care conditions (pooled OR $=1.67,95 \%$ CI $1.14-$ 2.44), although heterogeneity was present $(Q=24.60$, $\left.P<0.01 ; \mathrm{I}^{2}=59.35\right)$.

Trials included in the primary meta-analysis that provided brief or no contact ( $\leq 5$ minutes), when pooled, had a smaller OR than those providing extended contact (>5 minutes) (brief $\mathrm{OR}=1.49, \quad 95 \% \quad \mathrm{CI}$ 0.85-2.62; extended OR $=2.19$, 95\% CI 1.37-3.48); however, there was no statistically significant difference between the subgroups (relative OR $=1.47$, 95\% CI 0.71-3.06).

\section{Primary focus: sensitivity analyses and investigation of heterogeneity}

The removal of borderline trials [20,21,25-27] had minimal impact on the primary meta-analysis (pooled $\mathrm{OR}=1.99,95 \%$ CI 1.22-3.26), but heterogeneity was reduced considerably $\left(Q=8.29, P=0.22 ; I^{2}=27.6 \%\right)$. To investigate the source of heterogeneity in greater depth, two post-hoc sensitivity analyses were carried out. For the first, trials with intervention contact-based borderline characteristics were removed [20,25-27], revealing a slight reduction in the pooled OR with statistical significance becoming borderline (pooled OR $=1.69$, 95\% CI 0.99-2.87), although heterogeneity is still present $\left(\mathrm{Q}=18.52, \quad \mathrm{P}=0.01 ; \mathrm{I}^{2}=62.2 \%\right)$. However, when Moore et al.'s trial [21] is removed from the analysis only (pooled OR $=1.99$, 95\% CI 1.42-2.80), as a trial including baseline quitters in the sample, heterogeneity is reduced substantially $\left(\mathrm{Q}=13.93, \mathrm{P}=0.08 ; \mathrm{I}^{2}=28.2 \%\right)$, suggesting that this trial is a major source of the heterogeneity.

Sensitivity analyses indicated that the ICC approximation used for Lawrence et al.'s trial [20] was robust (high ICC 0.1-pooled OR $=1.81,95 \%$ CI 1.22-2.70; low ICC 0.01 -pooled $\mathrm{OR}=1.84,95 \%$ CI $1.23-2.74)$. Little change was observed when the two trials included in the primary meta-analysis that did not validate smoking status biochemically were removed (pooled $\mathrm{OR}=1.85$, 95\% CI 1.20-2.86). 


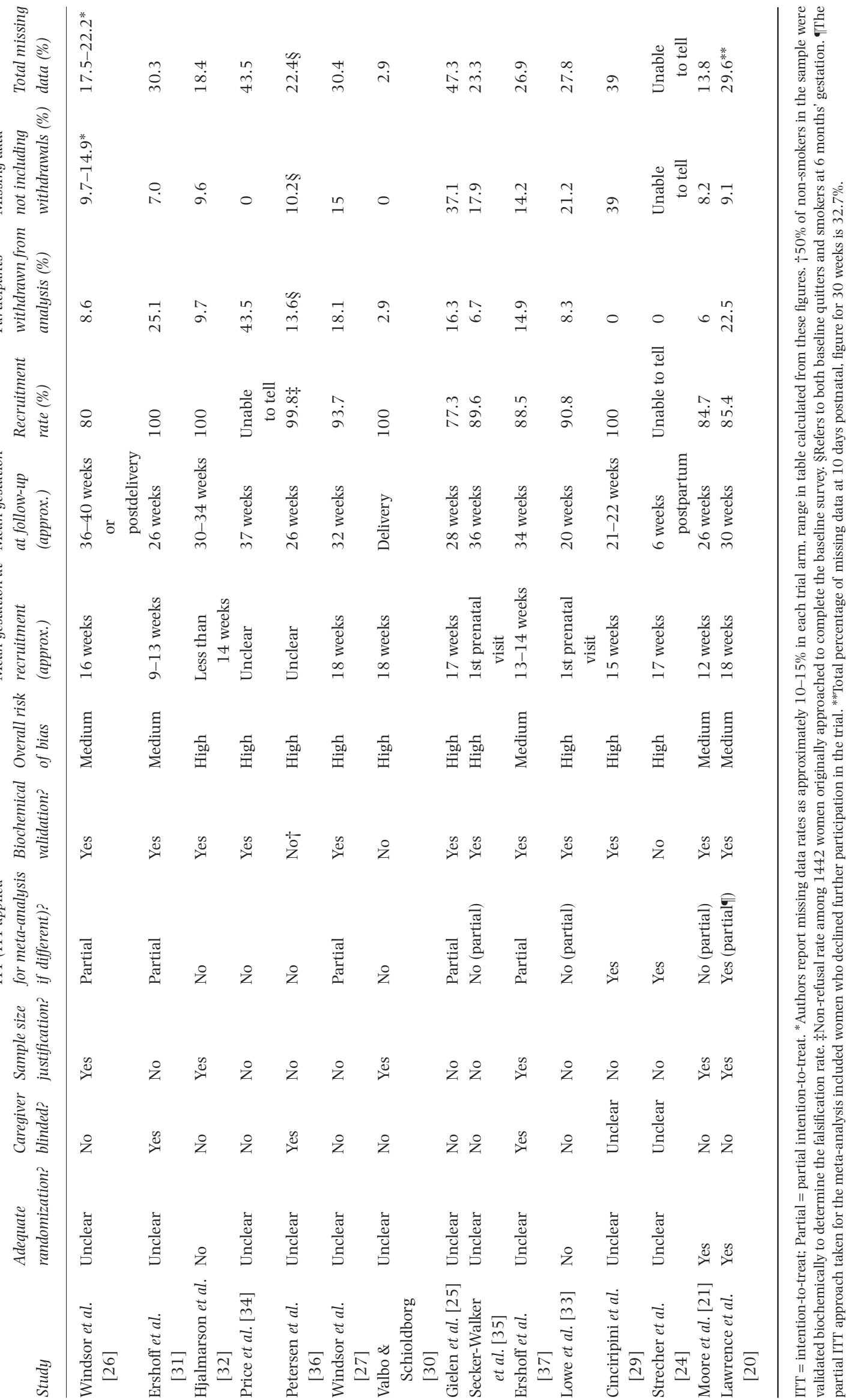




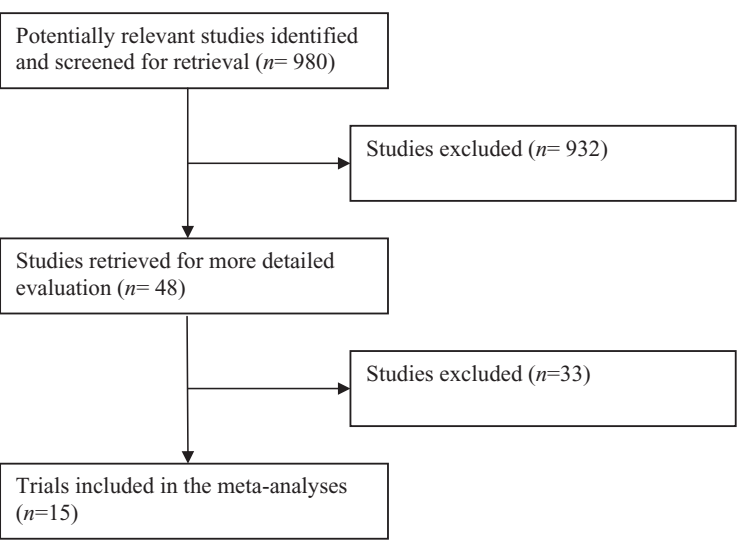

Figure I Review trial flow

Due to uncertainty, a post-hoc sensitivity analysis was carried out in the subgroup comparison analysis reclassifying Valbo \& Schioldborg's trial [30] as providing brief contact ( $\leq 5$ minutes). This reclassification caused some degree of change in the OR of each subgroup (brief $\mathrm{OR}=1.71,95 \%$ CI 0.97-3.03; extended OR $=2.01,95 \%$ CI 1.23-3.29), but no important change in the relative OR between subgroups.

Secondary focus: self-help versus more intensive self-help

A small difference was revealed among trials comparing arms of differing self-help material intensity favouring the more intensive arms, but the confidence intervals indicated a non-significant effect (pooled OR $=1.25,95 \%$ CI 0.81-1.94) (Fig. 2). Heterogeneity did not appear to be present $\left(Q=8.33, P=0.22 ; I^{2}=27.9 \%\right)$.

A subgroup analysis found no difference between non-tailored self-help interventions and self-help interventions tailored to participant characteristics (pooled $\mathrm{OR}=0.95,95 \%$ CI 0.60-1.48), with little heterogeneity between trials $\left(\mathrm{Q}=2.0, P=0.37 ; \mathrm{I}^{2}=0 \%\right)$.

\section{Secondary focus: sensitivity analyses}

The removal of Strecher et al.'s trial [24] in the secondary meta-analysis, due to the inclusion of baseline quitters in their sample and reliance on self-report, made little change to the findings (pooled OR $=1.31$, 95\% CI 0.782.22). Similarly, a post-hoc sensitivity analysis removing Cinciripini et al.'s trial [29] from the meta-analysis, due to the difficulty in determining whether their control materials should be classified as self-help, had little effect on the results (pooled OR $=1.34,95 \%$ CI 0.85-2.13).

\section{Inter-rater reliability}

Levels of agreement between reviewers for the quality criteria were adequate, with kappas of 0.47 for randomization, 0.76 for blinding, 1.0 for sample size calculation,
0.60 for intention-to-treat and 0.76 for biochemical validation. For outcome data and sample characteristics, percentage rates of agreement were $98 \%$ and $92 \%$, respectively.

\section{Process evaluation}

Ten of the 15 trials carried out some form of process evaluation, although there was little similarity in the data collected, making comparisons difficult. In general, the majority of participants used the materials to some degree, although the more sophisticated interventions experienced lower use rates. Ratings of usefulness were relatively high.

\section{DISCUSSION}

This review examined whether self-help interventions for pregnant smokers increase cessation rates over usual care and whether different self-help approaches are associated with different intervention efficacy.

\section{Summary of main results}

The primary meta-analysis provides evidence that selfhelp interventions for pregnant smokers increase cessation over usual care, with close to a doubling of the odds of quitting for those receiving a self-help intervention compared with those receiving usual care. This finding was relatively robust to small changes in inclusion/ exclusion criteria.

Further analyses found that self-help booklets increased quitting over usual care and, based on indirect comparisons, that face-to-face contact above 5 minutes did not increase intervention efficacy significantly. However, reliance on limited descriptions of contact and insufficient power to detect a difference between subgroups may be an issue with this latter analysis, especially given the notable change when one trial was re-categorized. One potential confounder in this latter analysis is that interventions providing a greater amount of face-to-face contact time tended to supply more intensive materials, which may account in part for the trend observed favouring the extended contact subgroup. Trials that systematically vary the quantity of intervention face-to-face contact need to be conducted in order to address this question adequately.

The secondary meta-analysis found that self-help materials of a greater intensity were not significantly more efficacious than those of lower intensity. However, given that the expected effect size between interventions of differing intensity would be small, the analysis may have been underpowered. In addition, tailored self-help interventions were found to be no more efficacious than non-tailored self-help interventions. However, only three 


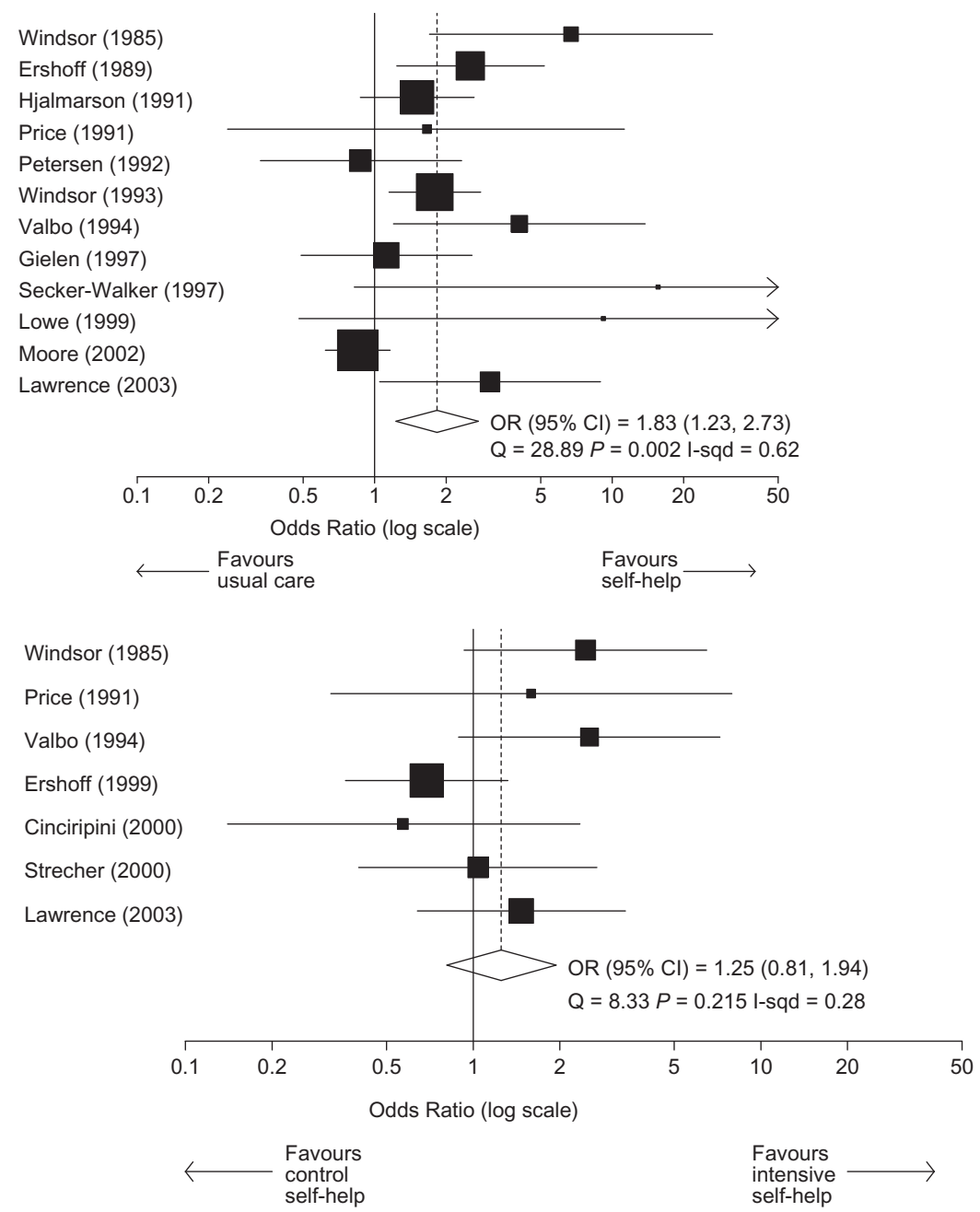

Figure 2 Primary meta-analysis (random effects) comparing usual care and self-help arm quit rates ( 12 trials) and secondary meta-analysis (random effects) comparing self-help and more intensive self-help arm quit rates (seven trials). trials were included in the tailoring subgroup analysis, one of which reported very low intervention compliance rates [37].

Interestingly, one trial was identified as accounting for much of the heterogeneity observed in the primary metaanalysis [21]. This may have been due in part to the disproportionately greater number of baseline quitters in the control group compared with the experimental group (30.1\% versus $25.1 \%$ ). When the authors of this trial adjusted their analyses for baseline smoking rates, as an attempt to control for smoking status, they found that the direction of effect moved from favouring the control group to favouring the experimental group, although neither effect was close to statistical significance. The findings are puzzling, given that $72 \%$ of the sample reported reading the materials and finding them useful, although without separate data for baseline quitters and smokers it is impossible to establish the impact of the intervention on smokers only.

While five trials could be described as being of high methodological quality, using Lumley et al.'s [14] criteria the remaining studies were of lower quality. There was no evidence of any detection bias and attrition bias was minimized, as a partial intention-to-treat approach was adopted for most trials. However, the possibility of performance and selection bias cannot be ruled out and so is a limitation of the review

\section{Internal and external validity of the evidence}

Internal validity of the review is fairly high as all trials, except one [29], were carried out alongside routine practice, and so samples, methods, interventions and intervention delivery environments were fairly similar across trials, and all but three trials used biochemical validation techniques to verify smoking status. However, there was some variation in follow-up time-points and length of time between baseline and follow-up, which may reduce the comparability of the trials.

In terms of external validity, the rates of recruitment into the trials were relatively high, with a median rate of $90.8 \%$ across the 13 of 15 trials reporting participation rates. With the exception of Cinciripini et al. [29], who recruited only women who were willing to set a quit date, 
the participants included in the meta-analyses could be considered to closely represent pregnant smokers who attend antenatal services in western countries. Additionally, Lawrence et al. [20] found no significant difference in 'stage of change' between women who participated and those who refused. However, as only one trial reported assessing the difference in motivation to quit between refusers and participants, it is possible that a difference existed. It should also be acknowledged that participants' behaviour may have been influenced by their awareness of taking part in research [40]. Despite these issues, we judge the ecological validity of the review to be high.

\section{Potential biases in the review process}

The process of trial selection relied on authors' descriptions of interventions and was, to some extent, subjective. This potential source of bias was minimized as the selection procedure was carried out independently by two reviewers, authors were contacted where possible and sensitivity analyses were carried out where there were uncertainties.

Determining the constitution of intervention materials was potentially problematic, as was defining the point at which a 'brief' leaflet becomes a self-help manual. However, in only one trial were the control materials difficult to classify [29], and a sensitivity analysis indicated that this trial was unlikely to be introducing bias into the secondary meta-analysis.

Gaining access to unpublished studies is difficult and there are no systematic methods for doing so. A generated funnel plot raised the possibility of bias, although when based on a small number of trials the results should be treated with considerable caution [41].

\section{Consistency with the smoking cessation literature}

The primary finding from this review corresponds closely with Lumley et al.'s primary finding and those of other reviews assessing a variety of smoking cessation interventions for pregnant smokers [13-15]. In addition, the finding that face-to-face contact levels is not associated with intervention efficacy is consistent with Kelley et al. and more general self-help reviews [13,42,43]. Similar to Lumley et al.'s [14] finding that the intensity of an intervention did not significantly increase its effectiveness, no significant additional benefit was found for self-help materials of greater intensity, although a trend in this direction was observed. Equally, the findings from our secondary and self-help booklet subgroup analyses correspond with Lancaster \& Stead's [7] qualitative assessment of different self-help materials for non-pregnant smokers, which found that only one of the 10 trials included in the assessment reported a difference in effect between different types of materials.
Our finding of no benefit for tailored over non-tailored self-help interventions does not match the findings from the non-pregnant smoking literature, which supports the efficacy of tailoring over non-tailoring [7,44-46]. However, with just three tailoring trials identified, it is clear that more trials are required to address this question adequately.

\section{Implications for practice}

A brief quitting message, as usually given in routine care with the provision of self-help materials supplied by a health professional, although relatively ineffective at persuading pregnant smokers to quit, is more effective than usual care and may be as effective as more resource intensive behavioural interventions.

Although many women expect and wish for information and advice from their midwives about smoking [47], advice perceived as being authoritarian or judgemental can have a negative impact $[48,49]$. Self-help materials may be attractive and helpful to some pregnant smokers because they allow them to digest smoking information and advice in their own time outside the clinical interaction, and avoid what might be seen as the potential for confrontation or judgement [6]. Alternatively, some pregnant smokers unable to quit unaided are able to do so with additional support, whether through low-intensity support such as self-help or more intensive support such as counselling. This may then leave a majority, most probably the more dependent smokers, who are either unmotivated to quit or who are unable to do so even with intensive behavioural support. This assertion is supported by the fact that all six trials included in the review that reported a subgroup assessment found that those who had quit during the study period were lighter smokers than those who continued to smoke. Self-help approaches should remain a pragmatic component in cost-effective best practice recommendations, although more effective approaches are likely to be required for heavier smokers.

\section{Implications for future research}

A disadvantage of most self-help materials is that they tend to be fairly generic, unlike an individualized counselling approach. This limitation might be reduced with greater investment in tailored approaches, which have been found to increase the use of materials, recall of information and behaviour change [50,51]. Future studies in this area should adopt high-quality research designs and strongly consider following-up participants postpartum and using continuous abstinence measures to assess longer-term quit rates. Additionally, a greater use of theory to underpin interventions is necessary in order to develop our theoretical understanding of prena- 
tal smoking beyond the stages of change concept, which has proved so far to be ineffective when guiding interventions for pregnant smokers [14].

It is equally important to explore the feasibility and potential of using alternative methods and modes of intervention delivery. Mobile telephone text messaging offers a potentially suitable and attractive medium to deliver smoking cessation interventions, and findings from research with non-pregnant smokers are promising [52].

\section{Conclusion}

Self-help interventions appear to be more effective than standard care and, given that they are relatively low cost, they are good value for money [27]. However, overall effectiveness is low and wide confidence intervals highlight the need for more evidence to increase our confidence that self-help materials are of value and to determine whether increased material intensity, tailoring of materials or increased face-to-face contact levels lead to improved intervention effectiveness.

\section{Acknowledgements}

The authors gratefully acknowledge the advice given by Dr Hazel Gilbert and Dr Tim Coleman on the preparation of this manuscript. This work was supported by Cancer Research UK (CR-UK) grant number C1345/A5809.

\section{References}

1. Castles A., Adams E. K., Melvin C. L., Kelsch C., Boulton M. L. Effects of smoking during pregnancy. Five meta-analyses. Am J Prev Med 1999; 16: 208-15.

2. Cnattingius $\mathrm{S}$. The epidemiology of smoking during pregnancy: smoking prevalence, maternal characteristics, and pregnancy outcomes. Nicotine Tob Res 2004; 6: S125-40.

3. Bolling K. Infant Feeding Survey 2005: Early Results. London: The Information Centre; 2006.

4. Owen L., McNeill A., Callum C. Trends in smoking during pregnancy in England, 1992-7: quota sampling surveys. BMJ 1998; 317: 728.

5. Taylor T., Hajek P. Smoking Cessation Services for Pregnant Women. London: Health Development Agency; 2001.

6. Ussher M., Etter J. F., West R. Perceived barriers to and benefits of attending a stop smoking course during pregnancy. Patient Educ Couns 2005; 61: 467-72.

7. Lancaster T., Stead L. Self-help interventions for smoking cessation. Cochrane Database Syst Rev 2005; Issue 3: CD001118.

8. Dijkstra A., De Vries H. The development of computergenerated tailored interventions. Patient Educ Couns 1999; 36: 193-203.

9. Consensus Workshop on Smoking Cessation During Pregnancy. Sponsored by the Robert Wood Johnson Foundation and the Smoke-Free Families Program in Collaboration with the Health Resources and Services Administration and the Centres for Disease Control and Prevention. Rockville, MD, 9-10 April 1998.
10. Ussher M., West R., Hibbs N. A survey of pregnant smokers' interest in different types of smoking cessation support. Patient Educ Couns 2004; 54: 67-72.

11. Hajek P., West R., Lee A., Foulds J., Owen L., Eiser J. R. et al. Randomized controlled trial of a midwife-delivered brief smoking cessation intervention in pregnancy. Addiction 2001; 96: 485-94.

12. Wisborg K., Henriksen T. B., Secher N. J. A prospective intervention study of stopping smoking in pregnancy in a routine antenatal care setting. Br J Obstet Gynaecol 1998; 105: 1171-6.

13. Kelley K., Bond R., Abraham C. Effective approaches to persuading pregnant women to quit smoking: a meta-analysis of intervention evaluation studies. Br J Health Psychol 2001; 6: 207-28.

14. Lumley J., Oliver S. S., Chamberlain C., Oakley L. Interventions for promoting smoking cessation during pregnancy. Cochrane Database Syst Rev 2004; Issue 4: CD001055.

15. Mullen P. D. Maternal smoking during pregnancy and evidence-based intervention to promote cessation. Prim Care 1999; 26: 577-89.

16. Dickersin K., Scherer R., Lefebvre C. Identifying relevant studies for systematic reviews. BMJ 1994; 309: 1286-91.

17. McMaster University. Search Strategies for EMBASE and PsycINFO in OVID Syntax. Available at: http:// hiru.mcmaster.ca/hedges/ 9 September 2005 (accessed 2 February 2006).

18. Clarke M., Oxman A. D. Cochrane Reviewer's Handbook 4.1.5 [updated April 2002]. The Cochrane Library, Issue 3, 2002. Oxford: Update Software; 2002.

19. Deeks J. J., Altman D. G. Effect measures for meta-analysis of trials with binary outcomes. In: Egger M., Smith G. D., Altman D. G., editors. Systematic Reviews in Healthcare. MetaAnalysis in Context. London: BMJ Books; 2001, p. 313-35.

20. Lawrence T., Aveyard P., Evans O., Cheng K. K. A cluster randomised controlled trial of smoking cessation in pregnant women comparing interventions based on the transtheoretical (stages of change) model to standard care. Tob Control 2003; 12: 168-77.

21. Moore L., Campbell R., Whelan A., Mills N., Lupton P., Misselbrook E. et al. Self help smoking cessation in pregnancy: cluster randomised controlled trial. BMJ 2002; 325: 1383.

22. Donner A., Klar N. Issues in the meta-analysis of cluster randomized trials. Stat Med 2002; 21: 2971-80.

23. Higgins J. P., Thompson S. G., Deeks J. J., Altman D. G. Measuring inconsistency in meta-analyses. BMJ 2003; 327: 557-60.

24. Strecher V. J., Bishop K. R., Bernhardt J., Thorp J. M., Cheuvront B., Potts P. Quit for keeps: tailored smoking cessation guides for pregnancy and beyond. Tob Control 2000; 9: III78-9.

25. Gielen A. C., Windsor R., Faden R. R., O'Campo P., Repke J., Davis M. Evaluation of a smoking cessation intervention for pregnant women in an urban prenatal clinic. Health Educ Res 1997; 12: 247-54.

26. Windsor R. A., Cutter G., Morris J., Reese Y., Manzella B., Bartlett E. E. et al. The effectiveness of smoking cessation methods for smokers in public health maternity clinics: a randomized trial. Am J Public Health 1985; 75: 1389-92.

27. Windsor R. A., Lowe J. B., Perkins L. L., Smith-Yoder D., Artz L., Crawford M. et al. Health education for pregnant smokers: its behavioral impact and cost benefit. Am J Public Health 1993; 83: 201-6.

28. Altman D., Ashby D., Birks J., Borenstein M., Campbell M., 
Deeks J. et al. Analysing and presenting results. In: Higgins J. P. T., Green S., editors. Cochrane Handbook for Systematic Reviews of Interventions 4.2.5 [updated May 2005]; Section 8. In: The Cochrane Library, Issue 3, 2005. Chichester: John Wiley \& Sons, Ltd; 2005, p. 97-166.

29. Cinciripini P. M., McClure J. B., Wetter D. W., Perry J., Blalock J. A., Cinciripini L. G. et al. An evaluation of videotaped vignettes for smoking cessation and relapse prevention during pregnancy: the very important pregnant smokers (VIPS) program. Tob Control 2000; 9: III61-3.

30. Valbo A., Schioldborg P. Smoking cessation in pregnancy: the effect of self-help manuals. J Matern Fetal Invest 1994; 4: $167-70$.

31. ErshoffD. H., Mullen P. D., Quinn V. P. A randomized trial of a serialized self-help smoking cessation program for pregnant women in an HMO. Am J Public Health 1989; 79: $182-7$.

32. Hjalmarson A. I., Hahn L., Svanberg B. Stopping smoking in pregnancy: effect of a self-help manual in controlled trial. $\mathrm{Br}$ J Obstet Gynaecol 1991; 98: 260-4.

33. Lowe J. B., Balanda K. P., Clare G. Evaluation of antenatal smoking cessation programs for pregnant women. Aust NZ J Public Health 1998; 22: 55-9.

34. Price J. H., Krol R. A., Desmond S. M., Losh D. P., Roberts S. M., Snyder F. F. Comparison of three antismoking interventions among pregnant women in an urban setting: a randomized trial. Psychol Rep 1991; 68: 595-604.

35. Secker-Walker R. H., Solomon L. J., Geller B. M., Flynn B. S., Worden J. K., Skelly J. M. et al. Modeling smoking cessation: exploring the use of a videotape to help pregnant women quit smoking. Women Health 1997; 25: 23-35.

36. Petersen L., Handel J., Kotch J., Podedworny T., Rosen A. Smoking reduction during pregnancy by a program of selfhelp and clinical support. Obstet Gynecol 1992; 79: 924-30.

37. Ershoff D. H., Quinn V. P., Boyd N. R., Stern J., Gregory M., Wirtschafter D. The Kaiser Permanente prenatal smokingcessation trial: when more isn't better, what is enough? Am J Prev Med 1999; 17: 161-8.

38. Prochaska J. O., Diclemente C. C. Stages and processes of self-change of smoking: toward an integrative model of change. J Consult Clin Psychol 1983; 51: 390-5.

39. Juni P., Altman D. G., Egger M. Assessing the quality of randomised controlled trials. In: Egger M., Smith G. D.,
Altman D. G., editors. Systematic Reviews in Healthcare. Meta-Analysis in Context. London: BMJ Books; 2001, p. 87108.

40. Franke R. H., Kaul J. D. The Hawthorne experiments: first statistical interpretation. Am Soc Rev 1978; 43: 623-43.

41. Egger M., Davey-Smith G., Schneider M., Minder C. Bias in meta-analysis detected by a simple, graphical test. BMJ 1997; 315: 629-34.

42. Gould R. A., Clum G. A. A meta-analysis of self-help treatment approaches. Clin Psychol Rev 1993; 13: 169-86.

43. Marrs R. W. A meta-analysis of bibliotherapy studies. Am J Community Psychol 1995; 23: 843-70.

44. Borland R., Balmford J., Hunt D. The effectiveness of personally tailored computer-generated advice letters for smoking cessation. Addiction 2004; 99: 369-77.

45. Prochaska J. O., DiClemente C. C., Velicer W. F., Rossi J. S. Standardized, individualized, interactive, and personalized self-help programs for smoking cessation. Health Psychol 1993; 12: 399-405.

46. Strecher V. J., Kreuter M., Den Boer D. J., Kobrin S., Hospers H. J., Skinner C. S. The effects of computer-tailored smoking cessation messages in family practice settings. J Fam Pract 1994; 39: 262-70.

47. Lendahls L., Ohman L., Liljestrand J., Hakansson A. Women's experiences of smoking during and after pregnancy as ascertained two to three years after birth. Midwifery 2002; 18: 214-22.

48. Arborelius E., Nyberg K. How should midwives discuss smoking behaviour in pregnancy with women of low educational attainment? Midwifery 1997; 13: 210-15.

49. Tod A. M. Barriers to smoking cessation in pregnancy: a qualitative study. Br J Commun Nurs 2003; 8: 56-64.

50. De Vries H., Brug J. Computer-tailored interventions motivating people to adopt health promoting behaviours: introduction to a new approach. Patient Educ Couns 1999; 36: 99-105.

51. Skinner C. S., Campbell M. K., Rimer B. K., Curry S., Prochaska J. O. How effective is tailored print communication? Ann Behav Med 1999; 21: 290-8.

52. Rodgers A., Corbett T., Bramley D., Riddell T., Wills M., Lin R. B. et al. Do u smoke after txt? Results of a randomised trial of smoking cessation using mobile phone text messaging. Tob Control 2005; 14: 255-61. 